\title{
Estudo comparativo de eficácia e segurança entre propofol e midazolam durante sedação para colonoscopia
}

\section{Comparative study of efficacy and safety between propofol and midazolam for sedation during colonoscopy}

\author{
Flávio Heuta Ivano ; Paula Christina Marra Romeiro; Jorge Eduardo Fouto Matias ${ }^{3}$; Giorgio Alfredo Pedroso Baretta4; Antonio \\ Katsumi Kay ; Carlos Akio Sasaki ${ }^{5}$; Regina Nakamoto ${ }^{5}$; Elizabeth Milla Tambara ${ }^{6}$
}

\section{RE S U M O}

\begin{abstract}
Objetivo: Comparar a segurança e a eficácia do propofol com a do midazolam na sedação profunda durante colonoscopias. Métodos: Sessenta e seis pacientes foram submetidos à colonoscopias e estudados prospectivamente. Um total de 50 pacientes recebeu 3,25 mg. $\mathrm{kg}^{-1}$ de peso de propofol. No grupo controle de 16 pacientes foi administrado 2,05 mg. $\mathrm{kg}^{-1}$ de peso de midazolam. A dose de manutenção foi titulada de acordo com a necessidade. Os parâmetros cardiovasculares e respiratórios observados foram a saturação de oxigênio, pressão arterial sistólica e diastólica e frequência cardíaca. Após o procedimento foi realizado um questionário sobre intercorrências como dor, desconforto e satisfação após a colonoscopia, utilizando uma escala visual de zero a dez. Foi aplicado o teste $t$ de Student para a análise estatística. Resultados: A amostra foi similar com relação às variáveis idade, peso, sexo e condição física. Houve diferença estatística significativa para os parâmetros saturação de oxigênio do sangue e pressão arterial sistólica entre os dois grupos. Não houve diferença estatística significativa para os parâmetros pressão arterial diastólica e pulso. Apesar das diferenças nos parâmetros cardiovasculares e respiratórios, não houve repercussões hemodinâmicas significativas. Não houve diferença estatística no parâmetro dor e satisfação. Os pacientes que apresentaram agitação (25\%) no grupo midazolam, relataram mais desconforto $(p=0,038)$. Conclusão: As variações nos parâmetros cardiovasculares e respiratórios, mesmo com diferenças significativas entre os grupos, não causaram repercussões clínicas significativas nos dois grupos, caracterizando a segurança na sedação profunda. A sedação com midazolam ou propofol não está associada a níveis de dor e satisfação diferentes entre os dois grupos. O grupo midazolan referiu significativamente mais desconforto que o grupo propofol.
\end{abstract}

Descritores: Sedação profunda. Colonoscopia. Propofol. Midazolam.

\section{INTRODUÇÃO}

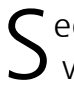
edação e analgesia na endoscopia gastrointestinal varia desde mínima sedação até anestesia geral e são utilizadas para reduzir a ansiedade do paciente frente ao exame, a dor, o desconforto e a lembrança do procedimento. Enquanto o exame endoscópico alto pode ser realizado sem dificuldade na ausência de sedação, muitos procedimentos endoscópicos altos e baixos necessitam de sedação moderada e analgesia, sendo denominadas de "sedação consciente". Nesta modalidade de sedação, o paciente é capaz de responder a estímulos verbais e táteis e os sistemas cardiovascular e respiratório permanecem ativos. Com sedação profunda, os pacientes respondem somente a estímulos dolorosos e o suporte ventilatório pode ser necessário. Na anestesia geral, eles não são responsivos a estímulos e o suporte ventilatório se impõe $e^{1,2}$.

Desde os anos 80, os benzodiazepínicos como o diazepam ou o midazolam têm sido utilizados como práti- ca rotineira. A associação de opióides, como a meperidina, com os benzodiazepínicos resulta em sinergismo com meIhor eficácia, entretanto, o aumento do tempo de latência e o prolongado período para recobrar a consciência é motivo de insatisfação ${ }^{3}$.

A droga ideal para sedação durante os procedimentos endoscópicos deve possuir as seguintes características: efeito imediato, sedação somente enquanto durar o procedimento, recuperação da consciência rapidamente, sem período residual de desordens mentais ou psicomotoras, e baixa incidência de efeitos colaterais. O uso rotineiro de uma droga de ação curta tem sido recomendado por muitos autores, sendo um meio para aumentar o conforto durante os procedimentos endoscópicos, resultando em procedimento de menor duração com um tempo de recuperação da consciência mais rápido 2,4-10.

O propofol (2,6-diisopropylphenol) é agente aprovado pelo Food and Drug Administration (FDA) dos USA, de ação extremamente curta no sistema nervoso central,

Trabalho realizado no Setor de Endoscopia Digestiva do Centro Médico-Hospitalar Sugisawa e no Programa de Pós-Graduação em Clínica Cirúrgica da Universidade Federal do Paraná -UFPR- Curitiba - PR- BR.

1. Doutorando do Programa de Pós-Graduação em Clínica Cirúrgica da Universidade Federal do Paraná - UFPR- Curitiba - PR- BR. 2. Aluna de Graduação do Curso de Medicina da UFPR- Curitiba - PR- BR. 3. Professor Adjunto do Departamento de Cirurgia da UFPR - Curitiba - PR- BR. 4. Doutorando do Programa de Pós-Graduação em Clínica Cirúrgica da UFPR- Curitiba - PR- BR. 5. Médicos Endoscopistas do Centro MédicoHospitalar Sugisawa, Curitiba-PR-BR. 6. Anestesiologista, Professora Adjunta do Departamento de Cirurgia da UFPR- Curitiba - PR- BR. 
classificado como droga que promove atividade do ácido gama aminobutírico (GABA) no cérebro, com propriedades sedativas e hipnóticas. Causa amnésia com mínima analgesia, utilizado para induzir e manter anestesia geral e na sedação profunda em endoscopias gastrointestinais, com ventilação assistida. Circulando ligada a uma proteína plasmática, tem rápido início de ação (30 a 60 s), é metabolizada no fígado, com "clearance" hepático de 2 a $4 \mathrm{~m}$, propiciando rápido recobrar da consciência e maior satisfação do paciente, em comparação com os sedativos

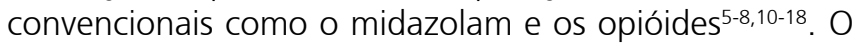
risco do paciente ser submetido a sedação profunda, associada à depressão respiratória, deve ser considerado.

Pelo fato de não existir antagonista para o propofol, os pacientes que são submetidos à sedação excessiva podem necessitar de ventilação assistida durante alguns minutos até a recuperação da consciência e da respiração espontânea. Por esse motivo, a administração de propofol requer treinamento específico e monitorização rigorosa, sendo recomendado que seja administrado por médicos anestesiologistas durante os procedimentos endoscópicos $6,8,12-14,17$.

O midazolam tem sido alvo de muitas queixas por parte dos pacientes e dos profissionais que executam esses exames em virtude da observação de sofrimento em decorrência do desconforto e da dor.

O objetivo deste trabalho foi avaliar comparativamente a sedação com midazolam e com propofol, em pacientes submetidos à colonoscopia, utilizando como parâmetros a eficácia e segurança, por meio dos efeitos respiratórios e cardiovasculares; a dor e o desconforto durante e após o procedimento; e a satisfação do paciente.

\section{MÉTODOS}

Trata-se de trabalho prospectivo desenvolvido no Setor de Endoscopia Digestiva do Centro Médico-Hospitalar Sugisawa (HS), com a colaboração do Serviço de Anestesiologia, aprovado pelo Comitê de Ética do mesmo hospital, com consentimento por escrito dos pacientes para sua inclusão no estudo.

Participaram dele 66 pacientes submetidos a procedimentos colonoscópicos, diagnósticos e terapêuticos, divididos em dois grupos, 50 sedados com propofol (grupo propofol) e 16 sedados com midazolam (grupo controle).

Foram incluídos nesta pesquisa pacientes de ambos os sexos que assinaram o termo de consentimento, com as seguintes características: faixa etária entre 16 e 75 anos; peso entre 50 e 100 kg; altura entre 1,50 e 1,90m; estado físico ASA I, II ou III de acordo com a American Society of Anesthesiologysts.

Foram utilizados como critério de exclusão: alergia a ovo ou soja e seus derivados (listados como contraindicados para a utilização de propofol); gravidez; pacientes com dificuldade de acesso a via aérea (incapacidade de abrir a boca, problemas na mandíbula, face ou pescoço); história prévia de apnéia do sono; desordens psi- quiátricas; pacientes classificados como ASA IV ou V; hipoxemia, hipotensão arterial, bradicardia e taquicardia previamente à administração das drogas.

O preparo de cólon iniciou dois dias antes do procedimento com dieta líquida sem resíduos e prescrição de picossulfatosódico 20 gotas de 8/8 h. Foi solicitado jejum absoluto de 6 a $8 \mathrm{~h}$ antes do procedimento. Recomendado o preparo de cólon com manitol - 500 mL, diluído em água ou suco de laranja - 500 mL, com início da ingestão oral a partir das 14 horas do dia anterior ao exame.

No grupo do propofol, a droga foi administrada por anestesiologista que monitorizou os dados vitais dos pacientes e os registrou, auxiliado por enfermeira e supervisionado pelo endoscopista. Um completo kit de ressuscitação cardiorrespiratório foi disponibilizado na sala de exame e na sala de recuperação pós-anestésica, conforme recomendação da Resolução do CFM 1670 de 2003. O paciente foi induzido com uma dose de 1 a $2 \mathrm{mg} \mathrm{kg}^{-1} \mathrm{de}$ propofol ( 2 mg. $\mathrm{kg}^{-1}$ para pacientes com idade inferior a 65 anos e $1 \mathrm{mg} \cdot \mathrm{kg}^{-1}$ para pacientes com idade superior a 65 anos) e quando necessário (paciente recuperando a consciência com movimentos ou apresentando reflexo palpebral), foi adicionado dose complementar. A dose foi adequada dependo da idade, peso, co-morbidades, uso contínuo de psicotrópico. O objetivo foi manter sedação profunda, com os pacientes incapazes de responder a comandos verbais ou resposta à estimulação táctil ou dolorosa.

A ausência de reflexo palpebral foi o sinal da adequada dose de indução da sedação. Os batimentos cardíacos, a saturação periférica de oxigênio arterial (SpO2) e a frequência respiratória foram monitorizados continuamente durante os procedimentos. A pressão arterial foi aferida antes da sedação, a cada três minutos durante o procedimento, imediatamente após o término e na sala de recuperação pós-anestésica. Foi utilizada suplementação de oxigênio (3 L/min via cateter nasal) em todos os pacientes antes, durante e após o procedimento. As doses iniciais para manter a sedação profunda foram administradas em intervalos menores, enquanto as doses subsequentes foram administradas com menos frequência.

No grupo controle (midazolam) a droga foi igualmente administrada por anestesiologista, com a mesma monitorização do grupo anterior, na dose de 0,1 mg. $\mathrm{kg}^{-1}$ de peso na indução e complementado conforme a necessidade (paciente recobrando a consciência com movimentos ou apresentando sinais de dor ou desconforto). Neste grupo os pacientes se mantiveram em sedação consciente, respondendo a comandos verbais e a estimulação tátil e dolorosa. Foram mantidos com suplementação de oxigênio $3 \mathrm{~L} / \mathrm{min}$.

A colonoscopia foi realizada com o paciente em decúbito lateral direito e movido para a posição de decúbito dorsal durante o procedimento, após a transposição do cólon descendente. O aparelho de colonoscopia foi introduzido através do canal anal, progrediu por todo o cólon até o ceco e foi introduzido na válvula íleo-cecal para o exame do íleo terminal. Durante o exame, foi observada toda 
mucosa colônica e do íleo terminal para detecção de formações polipóides, neoplásicas ou diverticulares. Procedimentos terapêuticos foram realizados em caso de necessidade.

Caracterizaram-se como complicações associadas à sedação: declínio da saturação de oxigênio no sangue arterial $\left(\mathrm{SpO}_{2}\right)$ a valores menores que $90 \%$, detectado pelo aparelho de oximetria digital, caracterizado como hipóxia; frequência cardíaca (FC) menor que 60 ou maior que 150 batimentos por minuto (bpm); pressão arterial sistólica (PAS) menor que $90 \mathrm{mmHg}$; e pressão arterial diastólica (PAD) menor que $50 \mathrm{mmHg}$. As complicações foram anotadas no protocolo pelo anestesiologista e pela enfermeira, revisado pelo colonoscopista.

Os pacientes foram transferidos para a sala de recuperação pós-anestésica (REPAI) quando os sinais vitais (PAS, PAD, FC e $\mathrm{SpO}_{2}$ ) estabilizaram-se. $\mathrm{Na}$ REPAI uma enfermeira realizou monitorização e, juntamente com o anestesiologista, determinou quando os pacientes estavam totalmente conscientes e capacitados para receber alta. Os dois grupos permaneceram em ambiente hospitalar por mais 60 minutos. Os sinais de recuperação consideraram nível de consciência, sinais vitais e função psicomotora por meio da observação de cinco categorias: atividade (capacidade de caminhar em linha reta sem instabilidade por cinco metros); condição respiratória (movimentos respiratórios sem esforço); estabilidade circulatória (variação de pressão de até $20 \%$ às medidas anteriores à sedação); oxigenação ( $\mathrm{SpO} 2$ superior a $92 \%$ em ar ambiente) e nível de consciência (totalmente acordado e respondendo a quesitos na REPAI). Os pacientes foram alertados, com antecedência, para não dirigir veículos automotores após o procedimento e para não utilizar transporte público desacompanhados.

Um questionário rápido sobre as intercorrências como dor, desconforto e satisfação durante o procedimento foi aplicado aos pacientes utilizando uma escala analógica visual de zero a dez. A variação da intensidade da dor foi de 0 = nenhuma dor e 10 = dor intensa; do desconforto, 0 = nenhum e 10 = muito desconforto e da satisfação com a sedação, 0 = insatisfeito e 10 = extremamente satisfeito ${ }^{15}$. O desconforto associado ao procedimento foi definido como distensão abdominal, náusea, cefaléia ou tontura. Os pacientes foram contatados após 24 horas e questionados sobre a presença de sinais de complicações e da manutenção dos escores da data do exame.

Todos os dados foram registrados e digitados em planilha eletrônica (Microsoft Excel®), conferidos e exportados para o programa Statistica®.

O teste estatístico aplicado foi o teste $t$ de Student para a diferença das médias com variâncias equivalentes. Foram testadas as hipóteses de que os parâmetros não possuem diferença após a aplicação dos sedativos midazolan e propofol. Logo, a hipótese testada é que a média com midazolam é similar a média com propofol (hipótese nula). O nível de significância adotado foi menor que $5 \%(p<0,05)$.

\section{RESULTADOS}

Os pacientes sedados com midazolam apresentaram média de idade de 48,56 anos (31 e 64 anos). Os sedados com propofol de 54,58 anos (31 a 72 anos).

O grupo do midazolam compôs-se de 37,5\% de pacientes do sexo masculino e o grupo do propofol de $42 \%$.

A dose média total utilizada de midazolam foi de 14,1 mg (0,205 mg. $\mathrm{kg}^{-1}$ de peso), com amplitude de 7,5 a $15 \mathrm{mg}\left(0,09\right.$ a 0,294 mg. $\mathrm{kg}^{-1}$ de peso) e a dose média de propofol, de $224 \mathrm{mg}$ (3,25 mg. $\mathrm{kg}^{-1}$ de peso), com amplitude de 100 a 400 mg (1,49 a 5,56 mg. $\mathrm{kg}^{-1}$ de peso).

A amostra de pacientes se mostrou similar com relação às variáveis como idade, peso, sexo e estado físico (ASA).

\section{Parâmetros Cardiovasculares e Respirató-}

rios

Nenhum paciente necessitou de intervenção emergencial (ventilação por máscara, intubação oro-traqueal ou procedimento de reanimação cardiorrespiratória).

Dos dezesseis pacientes submetidos à sedação com midazolam, nenhum desenvolveu hipoxemia. Dos 50 pacientes sob sedação com propofol, 18 pacientes (36\%) desenvolveram hipoxemia leve (SpO2 entre 90 e $85 \%$ ). Entretanto, responderam rapidamente à suplementação de oxigênio (3 a 6 litros/minuto). Houve diferença estatisticamente significativa no nível de oxigenação no sangue, com quadro de hipóxia discreta, SpO2 média de 96,7\%, para os sedados com midazolam e SpO2 médio de 94,5\%, para o grupo de propofol. Foram retirados da amostra os pacientes que apresentavam hipóxia previamente à sedação (um paciente do grupo midazolam e 12 pacientes do grupo propofol).

Houve dois casos $(12,5 \%)$ de hipotensão (PAS $<90 \mathrm{mmHg}$ ) nos pacientes sedados com midazolam e $16(32 \%)$ nos sedados com propofol.

Não houve casos de frequência cardíaca menor que 50 batimentos por minuto ( $\mathrm{FC}<50 \mathrm{bpm}$ ) nos sedados com midazolam. Houve dois casos (4\%) dos sedados com propofol, sem repercussões clínicas importantes.

As complicações como a depressão cardiovascular e respiratória apresentadas pelos pacientes foram relacionadas com os riscos baseados na classificação do American Society of Anesthesiologists (ASA).

Nos pacientes sedados com midazolam, 12 foram classificados como ASA I, sendo que destes, nenhum apresentou hipoxemia (SpO2<90\%), dois (12,5\%) apresentaram hipotensão (PAS $<90 \mathrm{mmHg}$ ) e nenhum bradicardia (FC $<50$ bpm). Nos classificados como ASA II (três pacientes) e ASA III (um paciente), nenhum apresentou hipoxemia (SpO2<90\%), hipotensão (PAS $<90 \mathrm{mmHg}$ ) ou bradicardia (FC<50bpm).

Nos que receberam sedação com propofol, 43 foram classificados como ASA I e destes, 13 (30\%) apresentaram hipoxemia (SpO2<90\%), 13 (26\%) hipotensão (PAS $<90 \mathrm{mmHg}$ ) e dois (4\%) bradicardia ( $\mathrm{FC}<50 \mathrm{bpm})$. Os classificados como ASA II (7 pacientes), três (6\%) apresen- 
taram hipoxemia (SpO2<90\%), três $(6 \%)$ hipotensão (PAS $<90 \mathrm{mmHg}$ ) e não se observou bradicardia ( $\mathrm{FC}<50$ bpm). Nenhum foi classificado como ASA III (Tabela 1).

Para os testes estatísticos das variáveis pressão arterial e frequência cardíaca, apenas um paciente foi excluído da amostra, do grupo do propofol, por apresentar hipotensão e bradicardia prévias.

Os pacientes foram testados estatisticamente para os parâmetros saturação de oxigênio no sangue (SpO2), pressão arterial (PA) e frequência cardíaca (FC), após a aplicação do sedativo. No caso da saturação de oxigênio, foram retirados da amostra os pacientes com quadro de hipoxemia previamente à sedação. Os resultados são demonstrado na tabela 2 .

Mesmo após a exclusão dos pacientes com hipoxemia previamente ao uso do sedativo, rejeitou-se a hipótese de que os níveis de saturação de oxigênio do sangue são iguais após o uso das drogas, sendo a média com midazolan significamente superior à média com propofol.

Para o parâmetro pressão arterial, os pacientes do grupo midazolan apresentaram em média pressão arterial sistólica superior aos pacientes do grupo propofol.

Não houve diferença estatisticamente significativa entre as pressões arteriais diastólicas após o uso do sedativo dos dois grupos.
Não houve diferença significativa na frequência cardíaca entre os pacientes sedados com midazolan, comparados aos sedados com propofol.

\section{Dor, Desconforto e Satisfação}

Resultados para os parâmetros de dor e desconforto durante a colonoscopia e satisfação após as colonoscopias, sedadas com midazolan, comparadas com propofol, com notas que variam de zero a dez, estão na tabela 3.

Não houve diferença no nível declarado de dor nos procedimentos de colonoscopia sedados com midazolan, comparados aos sedados com propofol.

Os pacientes do grupo midazolan apresentou mais desconforto durante as colonoscopias, que os do grupo propofol.

Não houve diferença significativa no parâmetro satisfação entre os grupos midazolan e propofol.

\section{DISCUSSÃO}

Embora recentes estudos tenham mostrado que o propofol é seguro quando administrado por enfermeiras treinadas com a supervisão do endoscopista, sem a super-

Tabela 1 - Frequência de complicações relacionados à condição física dos pacientes sedados com Midazolam e Propofol.

\begin{tabular}{lcccccccc}
\hline ASA & \multicolumn{2}{c}{ Pacientes } & \multicolumn{2}{c}{$\mathrm{SpO}_{2}<90 \%$} & \multicolumn{2}{c}{ PAS $<90 \mathrm{mmHg}$} & \multicolumn{2}{c}{$\mathrm{FC}<50 \mathrm{bpm}$} \\
& Midazolam & Propofol & Midazolam & Propofol & Midazolam & Propofol & Midazolam Propofol \\
\hline I & 12 & 43 & - & $15(30 \%)$ & $02(12,5 \%)$ & $13(26 \%)$ & - & $02(4 \%)$ \\
II & 03 & 07 & - & $03(6 \%)$ & - & $03(6 \%)$ & - & - \\
III & 01 & 00 & - & - & - & - & - & - \\
Total & 16 & 50 & - & $18(36 \%)$ & $02(12,5 \%)$ & $16(32 \%)$ & - \\
\hline
\end{tabular}

Tabela 2 - Teste estatístico t para os parâmetro saturação de oxigênio do sangue (SpO2), pressão arterial sistólica (PAS), pressão arterial diastólica (PAD) e frequência cardíaca (FC) para os pacientes submetidos à colonoscopias sedados com Midazolam, comparados aos sedados com Propofol.

\begin{tabular}{llclr}
\hline Grupos & \multicolumn{1}{c}{$\mathrm{SpO}_{2}$} & \multicolumn{1}{c}{ PAS } & PAD & FC \\
\hline Midazolam & $96,7 \%$ & $123,7 \mathrm{mmHg}$ & $79,2 \mathrm{mmHg}$ & $83,15 \mathrm{bpm}$ \\
Propofol & $94,5 \%$ & $113,6 \mathrm{mmHg}$ & $76,4 \mathrm{mmHG}$ & $89,74 \mathrm{bpm}$ \\
Estatística t & $-2,26(p=0.028)$ & $-2,05(p=0.044)$ & $-0,76(p=0.45)$ & $1,58(p=0.12)$ \\
\hline
\end{tabular}

Tabela 3 - Teste estatístico $t$ para os parâmetros dor, desconforto e satisfação durante as colonoscopias para os pacientes sedados com Midazolam, comparados aos sedados com Propofol.

\begin{tabular}{llll}
\hline Grupos & \multicolumn{1}{c}{ Dor } & Desconforto & Satisfação \\
\hline Midazolam & 1,06 & 2,81 & 9,31 \\
Propofol & 0,7 & 1,18 & 9,76 \\
Estatística t & $-0,52(p=0.37)$ & $-2,12(p=0.038)$ & $0,95(p=0.35)$ \\
\hline
\end{tabular}


visão de médicos anestesiologistas, muitos pacientes têm necessitado de ventilação assistida por máscara devido à apnéia prolongada 7,9,10,15,18-21.

Este estudo demonstra a experiência com segurança e ausência de desconforto de cinquenta pacientes submetidos à colonoscopia sob sedação profunda com propofol. Nenhum necessitou de intervenção emergencial (ventilação por máscara, intubação oro-traqueal ou procedimento de reanimação cardiorrespiratória). Dos 16 submetidos à colonoscopia com sedação com midazolam, nenhum paciente desenvolveu hipoxemia.

Dos que desenvolveram hipoxemia moderada, todos responderam rapidamente à inalação de oxigênio (3 $\mathrm{L} / \mathrm{m})$, nenhum desenvolveu hipoxemia importante. A saturação de oxigênio no sangue arterial no grupo do propofol apresentou quadro de hipoxemia com diferença estatisticamente significativa para o grupo midazolam. Apesar da diferença estatística, esta queda não trouxe comprometimento clínico importante, já que os pacientes permaneceram estáveis. Ao testar os efeitos dos dois medicamentos sobre a concentração de oxigênio no sangue, foram retirados da amostra os pacientes que apresentavam hipoxemia previamente à sedação, ou seja, hipoxemia relacionada à ansiedade ou sensibilidade do sensor digital do oxímetro. Nenhum paciente necessitou de intubação endotraqueal e ventilação mecânica ou por máscara com uma dose inicial menor de 0,25 a 0,5 mg. $\mathrm{kg}^{-1}$ de propofol, como corroborado no estudo de Kulling et al. ${ }^{20}$. Nos casos de hipoxemia descritos nos trabalhos da literatura optou-se por ventilação por máscara7,12,17,22.

Em relação à sedação de idosos, a literatura relata que o propofol pode ser usado com segurança e com boa tolerância. O trabalho de Heuss et al. ${ }^{23}$ sugere um cuidado maior na monitorização, em função da tendência de queda na saturação de oxigênio, e recomenda doses 10 a $20 \%$ menores. No seu estudo, os pacientes classificados como ASA I e Il apresentaram risco de evolução para hipóxia de $1,7 \%$, enquanto os ASA III e IV demonstraram aumento de risco de 3,6\%. SIPE et al. ${ }^{9}$ e Tohda et al. ${ }^{21}$ sugerem dose inicial reduzida para sedação efetiva e segura, com a metade da dose para pacientes com idade superior a 65 anos.

No presente estudo, o parâmetro saturação de oxigênio do sangue para os pacientes submetidos à colonoscopias sedados com midazolam, comparados aos sedados com propofol, mostrou diferença significativa com vantagem para o midazolam. Entretanto, sabe-se que esta diferença não causa repercussões clínicas. As vantagens do propofol são geralmente aceitas. Do ponto de vista anestésico, a sua dose adequada tem margem de segurança limítrofe, principalmente quando administrada por profissionais não anestesiologistas, particularmente no que se refere à depressão respiratória ${ }^{24}$

Dois casos $(12,5 \%)$ de hipotensão (PAS $<90 \mathrm{mmHg}$ ) foram observadas nas colonoscopias sedadas com midazolam e 16 casos (32\%), nas sedadas com propofol. Observou-se melhora espontânea, poucos foram tratados com infusão venosa de solução salina e não concorreram com repercussões clínicas significativas.
Mediante os dados anteriores, conclui-se que os casos de hipoxemia, hipotensão e bradicardia foram transitórios e responderam rapidamente à administração de oxigênio ou de solução salina, sem repercussões clínicas significativas.

A pressão arterial sistólica (PAS) para os sedados com midazolam, comparados aos sedados com propofol, mostra diferença significativa. Sabe-se, também, que esta diferença não causa repercussões hemodinâmicas.

Na situação caracterizada por excitação paradoxal após a administração de benzodiazepínico, a literatura cita a utilização de flumazenil25. Os resultados desta análise relacionados ao desconforto, corroboram o trabalho de Koshy et al. ${ }^{6}$, que comparou eficácia, segurança e recuperação dos pacientes sedados com propofol, aos sedados com a associação de midazolam e meperidina. A avaliação da recuperação demonstrou duas vezes mais conforto com propofol, que proporcionou sedação mais profunda, que a combinação de midazolam com meperidina. A recuperação do nível de consciência foi mais rápida, contudo sem significância estatística. Os parâmetros de segurança, como a pressão arterial, frequências cardíaca e respiratória e saturação de oxigênio, foram similares nos dois grupos analisados neste estudo.

Os grupo de Ulmer, Hansen e Rex, da Universidade de Indiana, publicaram muitos trabalhos sobre sedação com propofol em endoscopias e colonoscopias, administrados por enfermeiras treinadas, supervisionadas por endoscopistas ${ }^{26-28}$. Compararam o propofol com a associação de midazolam e fentanil e concluíram que o uso do propofol oferece inúmeras vantagens, como duração menor do exame, maior profundidade de sedação, recuperação da consciência mais rápida e alta mais precoce. O grupo sedado com propofol recebeu melhores escores dos testes de reflexos e de memória, porém o grau de satisfação foi similar entre os dois grupos, com o uso de escala visual analógica de zero a dez, como também observado neste estudo.

Ao contrário do trabalho de Weston et al. ${ }^{29}$ que comparou a utilização de propofol com a associação de midazolam e meperidina, administrados por enfermeiras treinadas em pacientes portadores de cirrose, o procedimento foi bem tolerado. Os pacientes manifestaram satisfação maior com a qualidade do exame e retornaram à condição prévia em melhores condições, quando comparados ao grupo midazolam associado à meperidina.

No estudo de Tohda et al. ${ }^{21}$, os pacientes realizaram exames sob sedação com associação de midazolam com meperidina e um novo exame com propofol. Foram questionados sobre a preferência do sedativo em próximo exame. A resposta foi de $85 \%$ para propofol e apenas 5\% para a associação d e midazolan/meperidina. O objetivo principal foi a satisfação do paciente, o tempo de recuperação do nível de consciência, avaliar a função neuropsíquica e as complicações. O tempo do procedimento foi menor para o grupo do propofol. Os pacientes que receberam sedação com propofol foram capazes permanecer em pé 
ao lado da maca, recobrar a consciência rapidamente e receber alta em período mais curto. Também demonstraram que houve grande satisfação após o procedimento e receberam escores melhores nos testes neuropsíquicos. Quatro pacientes do grupo midazolan/meperidina desenvolveram complicações menores e um paciente do grupo propofol desenvolveu desaturação importante e transitória de oxigênio abaixo de $85 \%$, fato não observado no neste trabalho. Estes resultados sugerem que o propofol causa maior satisfação, torna o procedimento mais rápido e talvez reduza os custos.

No trabalho de Hansen et al. ${ }^{26}$, sobre o desempenho técnico do colonoscopista em pacientes sedados com propofol por enfermeiras treinadas, o grupo propofol apresentou menor necessidade de mudança de decúbito, pressão sobre o abdômen de menor intensidade para a retificar o colonoscópio e o exame foi realizado em um tempo menor que o grupo sedado com midazolam associado a meperidina. Concluíram que a administração de propofol por enfermeiras é seguro e o procedimento foi simplificado, em comparação com o grupo midazolam associado à meperidina.

Segundo Bell ${ }^{23}$, porém, existe convicção de que o propofol é uma droga anestésica que deve ser administrada por anestesiologistas, com o que concordam Graber ${ }^{30}$ e Jung et al. ${ }^{14}$. Estes últimos, em 2000, compararam a qualidade da sedação de propofol (354 mg) com midazolam (8 mg) em colangiopancreatografia endoscópica retrógrada e julgaram que a qualidade da sedação é significativamente superior para o propofol e o tempo de recuperação do nível de consciência foi mais curto.
A avaliação comparativa da sedação com midazolam ou propofol em pacientes submetidos à colonoscopia permitiu concluir que o grupo que recebeu propofol apresentou redução estatisticamente significativa na saturação de oxigênio do sangue arterial e na pressão arterial sistólica, sem repercussões clínicas. Não houve hipoxemia intensa nos dois grupos. Não houve diferença significativa entre os dois grupos em relação à pressão arterial diastólica e à frequência cardíaca. Estes parâmetros caracterizam que o midazolam e o propofol são drogas seguras. A dor durante as colonoscopias foi de pequena intensidade nos dois grupos sem diferença significativa. O grupo midazolam referiu mais desconforto que o grupo propofol, principalmente levando em consideração o percentual elevado de pacientes que apresentaram agitação. O grau de satisfação foi elevado para os dois grupos, sem diferença significativa. Novos estudos poderiam dar continuidade em outras áreas da endoscopia, com associação de medicamentos ao propofol, para aprimoramento das condutas.

Em conclusão, os achados deste trabalho sugerem que o propofol e o midazolam são drogas seguras para sedação em colonoscopia, porém o midazolam apresentou maior desconforto que o propofol. As variações nos parâmetros cardiovasculares e respiratórios, mesmo com diferenças significativas entre os grupos, não causaram repercussões clínicas significativas nos dois grupos, caracterizando a segurança na sedação profunda. A sedação com midazolam ou propofol não está associada a níveis de dor e satisfação diferentes entre os dois grupos. O grupo midazolan referiu significativamente mais desconforto que o grupo propofol.

\section{A B S T R A C T}

Objective: To compare safety and efficacy of propofol with midazolam for deep sedation in the colonoscopy. Methods: In a prospective way, 66 patients underwent colonoscopy - 50 patients received propofol in an average dose of 3,25 mg. $\mathrm{kg}^{-1}$ and 16 patients (control group) received midazolam with total average dose of $2,05 \mathrm{mg} . \mathrm{kg}^{-1}$. The dose of medication was titrated according to patient need. The analyzed cardiovascular and respiratory parameters were: oxygen saturation, systolic and diastolic blood pressure and heart rate. After the colonoscopy, a survey with a visual scale from 0 to 10 and questions regarding the pain, discomfort and satisfaction was applied. The statistics analyzed by the t Student test. Results: Groups were similar regarding the age, weight, sex and physical conditions (ASA grade). None of the patient required emergencial treatment. None of the cardiovascular and respiratory parameters with statistics differences produced hemodynamic repercussions. The pain and satisfaction parameters showed no significant difference between the midazolam group and propofol group The discomfort parameter during the colonoscopic showed significant difference $(p=0,038)$ between the midazolam group (score 2,81) and propofol group (score 1,18) and all the patients wich had presented paradoxal excitation (25\%) in this group reported discomfort. Conclusion: The cardiovascular and respiratory parameters variation, even when different between groups, didn't produced clinical repercussions. The pain and satisfaction parameters showed no significant difference between groups. It was demonstrated that the midazolam group referred more discomfort than the propofol group.

Key words: Deep sedation. Colonoscopy. Propofol. Midazolam.

\section{REFERENCIAS}

1. American Society of Anesthesiologists Task Force on Sedation and Analgesia by Non-Anesthesiologists. Practice guidelines for sedation and analgesia by non anesthesiologists. Anesthesiology. 2002;96(4):1004-17.
2. Training Committee. American Society for Gastrointestinal Endoscopy. Training guideline for use of propofol in gastrointestinal endoscopy. Gastrointest Endosc. 2004;60(2):167-72.

3. Schutz SM, Lee JG, Schmitt CM, Almon M, Baillie J. Clues to patient dissatisfaction with conscious sedation for colonoscopy. Am J Gastroenterol. 1994;89(9):1476-9. 
4. Bhardwaj G, Conlon S, Bowles J, Baralt J. Use of midazolam and propofol during colonoscopy: 7 years of experience. Am J Gastroenterol. 2002;97(2):495-7.

5. Heuss LT, Schnieper P, Drewe J, Pflimlin E, Beglinger C. Conscious sedation with propofol in elderly patients: a prospective evaluation. Aliment Pharmacol Ther. 2003;17(12):1493-501.

6. Koshy G, Nair S, Norkus EP, Hertan HI, Pitchumoni CS. Propofol versus midazolam and meperidine for conscious sedation in $\mathrm{Gl}$ endoscopy. Am J Gastroenterol. 2000;95(6):1476-9.

7. Rex DK, Overley C, Kinser K, Coates M, Lee A, Goodwine BW et al. Safety of propofol administered by registered nurses with gastroenterologist supervision in 2000 endoscopic cases. Am J Gastroenterol. 2002;97(5):1159-63.

8. Seifert H, Schmitt TH, Gultekin T, Caspary WF, Wehrmann T. Sedation with propofol plus midazolam versus propofol alone for interventional endoscopic procedures: a prospective, randomized study. Aliment Pharmacol Ther. 2000;14(9): 1207-14.

9. Sipe BW, Rex DK, Latinovich D, Overley C, Kinser K, Bratcher L et al. Propofol versus midazolam/meperidine for outpatient colonoscopy: administration by nurses supervised by endoscopists. Gastrointest Endosc. 2002; 55(7): 815-25

10. Walker JA, Mcintyre RD, Schleinitz PF, Jacobson KN, Haulk AA, Adesman $P$ et al. Nurse-administered propofol sedation without anesthesia specialists in 9152 endoscopic cases in an ambulatory surgery center. Am J Gastroenterol. 2003;98(8): 1744-50.

11. Bryson HM, Fulton BR, Faulds D. Propofol. An update of its use in anaesthesia and conscious sedation. Drugs. 1995;50(3):513-59.

12. Carlsson U, Grattidge P. Sedation for upper gastrointestinal endoscopy: a comparative study of propofol and midazolam. Endoscopy. 1995; 27(3): 240-3.

13. Gillham MJ, Hutchinson RC, Carter R, Kenny GN. Patient maintained sedation for ERCP with a target-controlled infusion of propofol: a pilot study. Gastrointest Endosc. 2001; 54(1): 14-7.

14. Jung M, Hofmann C, Kiesslinch R, Brackertz A. Improved sedation in diagnostic and therapeutic ERCP: propofol is an alternative to midazolam. Endoscopy. 2000; 32(3):233-8.

15. Kulling D, Fantin AC, Biro P, Bauerfeind P, Fried M. Safer colonoscopy with patient-controlled analgesia and sedation with propofol and alfentanil. Gastrointest Endosc. 2001;54(1):1-7.

16. Nelson DB, Barkun AN, Block KP, Burdick JS, Ginsberg GG, Greenwald DA et al. Propofol use during gastrointestinal endoscopy. Gastrointest Endosc. 2001; 53(7): 876-9.

17. Ng JM, Kong CF, Nyam D. Patient-controlled sedation with propofol for colonoscopy. Gastrointest Endosc. 2001;54(1):8-13.

18. Vargo JJ, Zuccaro G, Dumot JA, Shermock KM, Morrow JB, Conwell $D L$ et al. Gastroenterologist-administered propofol versus meperidine and midazolam for advanced upper endoscopy: a prospective randomized trial. Gastroenterology. 2002; 123(1): 8-16.

19. Heuss LT, Schnieper P, Drewe J, Pflimlin E, Beglinger C. Risk stratification and safe administration of propofol by registered nurses supervised by the gastroenterologist: a prospective observational study of more than 2000 cases. Gastrointest Endosc. 2003;57(6):664-71
20. Kulling D, Rothenbuhler R, Inauen W. Safety of non anesthetist sedation with propofol for outpatient colonoscopy and esophagogastroduodenoscopy. Endoscopy. 2003;35(8):679-82.

21. Tohda G, Higashi S, Wakahara S, Morikawa M, Sakumoto $H_{\text {, }}$ Kane T. Propofol sedation during endoscopic procedures: safe and effective administration by registered nurses supervised by endoscopists. Endoscopy. 2006; 38(4):360-7.

22. Vargo JJ, Zuccaro G, Dumot JA, Shay SS, Conwell DL, Morrow JB. Gastroenterologist-administered propofol for therapeutic upper endoscopy with graphic assessment of respiratory activity: a case series. Gastrointest Endosc. 2000; 52(2):250-5.

23. Bell GD. Premedication, preparation, and surveillance. Endoscopy. 2002; 34(1): 2-12.

24. Bell GD, Charlton JE. Colonoscopy-is sedation necessary and is there any role for intravenous propofol? Endoscopy. 2000; 32(3):264-7.

25. Fulton SA, Mullen KD. Completion of upper endoscopic procedures despite paradoxical reaction to midazolam: a role for flumazenil? Am J Gastroenterol. 2000; 95(3):809-11.

26. Hansen JJ, Ulmer BJ, Rex DK. Technical performance of colonoscopy in patients sedated with nurse-administered propofol. Am J Gastroenterol. 2004;99(1):52-6.

27. Rex DK, Heuss LT, Walker JA, Qi R. Trained registered nurses/ endoscopy teams can administer propofol safely for endoscopy. Gastroenterology. 2005; 129(5):1384-91.

28. Ulmer BJ, Hansen JJ, Overley CA, Symms MR, Chadalawada V, Liangpunsakul $S$ et al. Propofol versus midazolam/fentanyl for outpatient colonoscopy: administration by nurses supervised by endoscopists. Clin Gastroenterol Hepatol. 2003; 1(6): 425-32.

29. Weston BR, Chadalawada V, Chalasani N, Kwo P, Overley CA, Symms $M$ et al. Nurse-administered propofol versus midazolam and meperidine for upper endoscopy in cirrhotic patients. Am J Gastroenterol. 2003; 98(11):2440-7.

30. Graber RG. Propofol in the endoscopy suite: an anesthesiologist's perspective. Gastrointest Endosc. 1999;49(6):803-6.

Recebido em 22/12/2008

Aceito para publicação em 23/02/2009

Conflito de interesse: nenhum

Fonte de financiamento: nenhuma

\section{Como citar este artigo:}

Ivano FH, Romeiro PCM, Matias JEF, Barretta GAP, Kay AK, Sasaki CA Nakamoto R, Tambara EM. Estudo comparativo de eficácia e segurança entre propofol e midazolam durante sedação para colonoscopia. Rev Col Bras Cir. [periódico na Internet] 2010; 37(1). Disponível em URL: http://www.scielo.br/rcbc

\section{Endereço para correspondência:}

Flávio Heuta Ivano

Rua General Carneiro, $181-7^{\circ}$ Andar Central - Hospital de Clínicas Centro

80060-900. - Curitiba - Paraná 